\title{
Proposal and Evaluation of Toilet Timing Suggestion Methods for the Elderly
}

\author{
Airi Tsuji \\ Graduate School \\ of Engineering and Science, \\ Kyoto Institute of Technology \\ Matsugasaki, Sakyo-ku, Kyoto \\ 606-8585, Japan
}

\author{
Tomoko Yonezawa \\ Kansai University \\ 2-1-1 Ryozenji, Takatsuki \\ Osaka 569-1095, Japan
}

\author{
HirotakeYamazoe \\ Osaka University \\ 1-31 Machikaneyama, Toyonaka \\ Osaka 567-0047, Japan
}

\author{
Shinji Abe Hiroshima \\ Institute of Technology 2-1-1 \\ Miyake,Saeki-ku Hiroshima \\ 731-5193 Japan
}

\author{
Noriaki Kuwahara \\ Graduate School \\ of Engineering and Science, \\ Kyoto Institute of Technology \\ Matsugasaki, Sakyo-ku, \\ Kyoto 606-8585, Japan
}

\author{
Kazunari Morimoto \\ Graduate School \\ of Engineering and Science, \\ Kyoto Institute of Technology \\ Matsugasaki, Sakyo-ku, \\ Kyoto 606-8585, Japan
}

\begin{abstract}
Elderly people need to urinate frequently, and when they go on outings they often have a difficult time finding restrooms. Because of this, researching a body water management system is needed. Our proposed system calculates timing trips to the toilet in consideration with both their schedules and the amount of body water needing to be expelled, and recommends using the restroom with sufficient time before needing to urinate. In this paper, we describe the suggested methods of this system and show the experimental results for the toilet timing suggestion methods.
\end{abstract}

Keywords-Elderly; Suggestion-method;

\section{INTRODUCTION}

For healthy elderly people, leaving their homes is one of the most important activities in preserving their cognitive and physical abilities; this also provides good mental stimulation and pleasure in their daily lives [1]. Promoting barrier-free environments in Japanese public spaces encourages the elderly to leave their homes without assistance. However, going out is likely to become difficult for some elderly people as they age, especially when managing their body water balances. Because elderly people often have weak bladders, they are likely to experience the need for frequent urination and often encounter difficult situations when searching for restrooms while controlling their bladders. If a caregiver accompanies the elderly person, the caregiver can suggest going to the restroom in sufficient time before the elderly person needs to use one, or can look for the nearest restroom for their patient. However, the number of the elderly living alone is rapidly increasing in Japan, and they cannot receive such support. Consequently, they often tend not to drink enough water during their outings due to their anxiety about using restrooms. This is a likely cause of dehydration, especially in the summer [2]. In order to aim for elderly people's comfortable and independent outing experiences, we have researched and developed toilet and drinking timing suggestion systems according to their outing schedules, their surrounding environments, and their activities like eating and drinking [3][4][5]. However, our calculation formulas regarding the amount of body water encountered some problems. Therefore, we have been trying to resolve these issues by researching notification methods. In order to improve our system, we have devised suggested methods of toilet timing for the elderly, trying to avoid interfering with their outing schedule while not ignoring trips to the restroom. In this paper we describe the experimental results when two toilet timing suggestion methods were evaluated.

\section{TOILET TIMING CALCULATION METHODS}

\section{A. Estimating the amount of body water}

According to previous research on water balance in the human body, we formulated a simplistic physiological formula for non-invasive estimation of the amount of body water; based on those estimations, times to drink fluids and use the toilet are calculated. According to previous studies, the total amount of voided volume of a well-rounded healthy elderly person in a day is assumed to be about $1500 \mathrm{ml}$, and the amount of the urine in the bladder when they feel the need to void is assumed to be $150 \mathrm{ml}$ [6][7]. We also assumed that the total amount of voided volume changes according to the increase of body surface area and body surface area correlates with body weight [8]. In a statistical survey of physical fitness and exercise abilities conducted by the Ministry of Education, Culture, Sports, Science and Technology, the average weight of Japanese adults was $65.15 \mathrm{~kg}$ for male and $53.04 \mathrm{~kg}$ for female [9]. Furthermore, we assume that that the total amount of voided volume changes according to the air temperature [10]; it increases or decreases by $0.6 \mathrm{ml} / 1 \mathrm{oC}$ from the baseline of 19.4oC. Consequently, we obtained the physiological formula 
of Eq. 1 and Eq. 2 for the non-invasive estimate of the amount of body water. The total amount of voided volume is divided by the length of hours spent awake (about 17 hours). The toilet timing is calculated by Eq.3.

$$
\begin{gathered}
\text { MaleU } \\
=1500+(W-65.15) \times 1.0-(T-19.4) \times 0.6 \\
\text { FemaleU } \\
=1500+(W-53.04) \times 1.0-(T-19.4) \times 0.6 \\
\text { ToiletInterval }=150 /(U / 17)
\end{gathered}
$$

$U$ is the total amount of excretion in one day[ml], Interval is the basic interval[hr], $T$ is outside temperature[degrees Celsius], $W$ is body weight[kg].

We set the prediction formula (Eq.4) to calculate the amount of sweat per day. The amount of sweat increases or decreases by $2.72 \mathrm{~g} / \mathrm{Body}$ weight $/ 1{ }^{\circ} \mathrm{C}$ when the air temperature is more than $16.5{ }^{\circ} \mathrm{C}$ [10].The amount of excretion per hour (Eq.5) considers the amount of voided urine and perspiration amounts $(9.1 \mathrm{ml})$. The appropriate drinking times (Eq.6) are derived from the risks of dehydration due to inadequate drink intake.

$$
\begin{gathered}
\text { Sweat } \\
=W \times(S 0+(T-19.4) \times 2.72) \\
\text { Drainage } \\
=(U / 17)+(\text { Sweat } / 17)+(9.1 \times W / 17)+(900 / 17) \\
\text { DrinkInterval }=(W \times 0.02) / \text { Drainage }
\end{gathered}
$$

$U$ is the total amount of excretion in one day[ml], Interval is the basic interval[hr], $T$ is outside temperature[degrees Celsius], $W$ is body weight $[\mathrm{kg}]$. SO the amount of sweating per body weight $[\mathrm{ml}]$.

\section{B. Rearranging Toilet Timing}

In order to maintain good health, adequate hydration is necessary. For this purpose, Japanese elderly people often carry water flasks when leaving their homes; they often have lunch or dinner with their friends or family during their outings. Such activities increase the overall amount of body water. Excess water in the body is excreted by the kidneys and passed into the bladder. We assume the amount of the water intake per meal is $200 \mathrm{ml}$, and recalculate the total amount of voided volume at each meal (Eq.7). Toilet timing is rearranged (Eq.3), with $\mathrm{U}$ representing the total amount of voided volume [ml].

$$
\operatorname{Drink} U=U+200
$$

$U$ is the total excretion of the day[ml].

\section{Problems Inherent in These Formulas}

We discovered some formulaic problems in previous studies[5]-for example, male subjects produced larger amounts of urine than $150 \mathrm{ml}$, and one male subject didn't receive the suggestion to use the toilet when he wanted. We are improving the formula. However, just improving the formula would not solve all problems. To address these problems, we created the following suggestion methods.

\section{Suggestion Methods}

In order to avoid interfering with their outing schedule and ignoring the suggestions, we devised two toilet timing suggestion methods.

\section{A. “Consideration” Strategy Situations}

The situation where the user can't go to the restroom easily often occur during daily activities. In order to prevent such situations, the system considers the user's schedule and avoids notifying the user during activities such as shopping, having lunch, or seeing a movie. Therefore, if the next toilet time is expected to be in the middle of an activity, the restroom time is adjusted to 5 minutes before the activity's start time. The system makes adjustments to the time calculated by Eq.1.

\section{B. "Step-by-Step” Suggestion Strategy}

Timing to use the restroom is a sensitive issue in general. If our proposed system unnecessarily recommends going to the restroom repeatedly, the user might feel that the system is annoying. Also, the suggestion might be ignored when the user is in the middle of their activities. Furthermore, the elderly are likely to have hearing difficulties; the verbal toileting suggestions might sometimes go unnoticed. Therefore, we created with the "step-by-step" suggestion of toilet timing. It consists of three types of suggestions depending on the urgency; "Recommend", "Notification", and "Alert". In order to explain the details of the suggestion algorithm as shown below we define two types of intervals; current interval Ic is calculated from the recent toilet timing using Eq.3, while the interval for suggestion is Im. Also, Ti denotes the elapsed time from the last restroom timing.

\section{Recommend}

When $\mathrm{Im} ; \mathrm{Ti} i \mathrm{Ic}$, because the situation is less urgent the system only recommends the subject to Notification use the restroom by both voice and text message.

When $\mathrm{Ic}=\mathrm{Ti}$, because the urgency increases to some extent, the system notifies the subject once more to use the restroom by both voice and text message.

Alert

After each suggestion as shown above, the system requires the user to confirm whether the user actually went to the restroom. If the user ignored both suggestions and a certain period of time elapses, due to the increase in urgency the system alerts the subject to use the restroom repeatedly by both voice and vibration.

\section{EXPERIMENTAL OVERVIEW}

In order to evaluate the effectiveness of the proposed suggestion methods we conducted the following experiments. Gauging the task achievement level and the delay time, tentative shopping tasks were set as shown below. We measured the 
TABLE I: Experimental Conditions of Suggestion Method

\begin{tabular}{lcc}
\hline Experimental conditions & Considering & Step by step \\
\hline No strategy & $\times$ & $\times$ \\
"Consideration" strategy & $\bigcirc$ & $\times$ \\
"Step-by-step" strategy & $\times$ & $\bigcirc$ \\
Using both strategies & $\bigcirc$ & $\bigcirc$ \\
\hline
\end{tabular}

delay from the suggestion to the action that the subject took, plus the task's end result. The experiments were conducted by comparing the four conditions as shown in Table II, incorporating the "Consideration" and "Step-by-Step" approaches. In this experiment, "Consideration" meant that the suggestion occurred before or after each shopping task; the "Step-byStep" intervals were 15 seconds and 10 seconds. The subjects performed trials under all conditions in random order. In order to investigate impressions of all conditions the subjects not only answered a questionnaire after each trial, but also answered a questionnaire at the end of the experiments to indicate which conditions were most preferable.

\section{A. Subjects}

The target users of our proposed system are elderly people. Therefore, the subjects were recruited from the age groups as shown in Table II.

\section{TABLE II: Subjects Overview}

\begin{tabular}{lrr}
\hline Group name & Number(male,female) & Age(ave) \\
\hline Subjects & $26(13,13)$ & $64-75(69.27)$ \\
\hline
\end{tabular}

\section{B. Experimental Setup}

The experimental setup is shown in Fig.1, and the setup simulating toilet timing suggestions during shopping is shown in Fig.2. Pushing the button while the subject sat on the available chair indicated that the subject went to the restroom. There were three places (A, B, and C) to pick up balls that simulated shopping item displays; the room also had a checkout counter. Colored cones and plastic tapes were used, indicating the walking path. Balls colored red, yellow and blue are shown in Fig.3; picking up the balls simulated buying goods. The subject was notified of the toilet timing suggestions by an audible cue emitted from a portable device.

\section{Experimental Scenario}

The experiment's scenario was as follows. Fig.1 shows the shopping task. The subject waited on the chair for a direction from the hand-held terminal. When the direction was suggested to the subject as shown below, s/he followed the instructions.

\section{Start Shopping Task}

A voice announced, "Start shopping" and "Take a red ball." In order to simulate shopping the subject carried the basket to locations A, B, and $\mathrm{C}$, picking up colored balls in accordance with the directions. Then the subject took their colored balls to the checkout basket. The subject continued shopping until the "End Shopping Task" was announced.

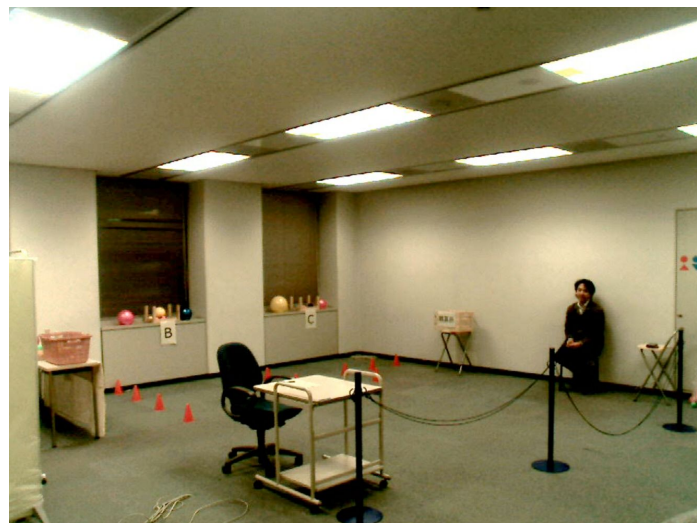

Fig. 2: Experimental Setup - Room View

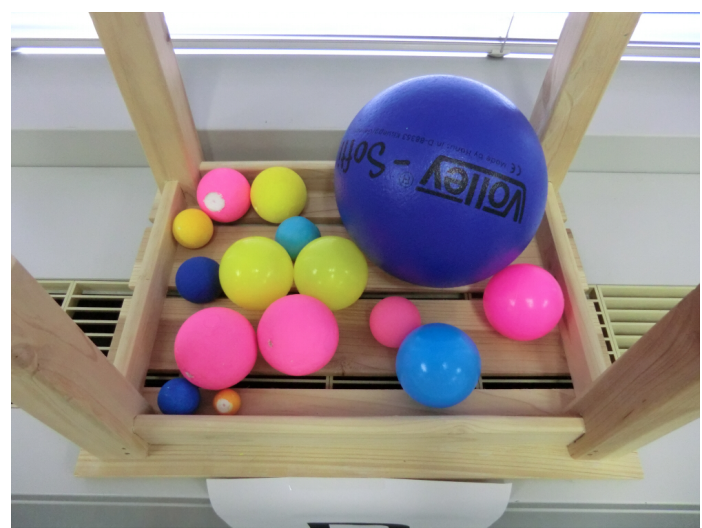

Fig. 3: Balls Used During the Experiment

\section{End Shopping Task}

"Stop shopping and return to the waiting chair" was announced. The subject returned the shopping basket to the start position and sat down on the available chair, waiting for the next suggestion.

\section{Suggestion to Use the Toilet}

First, a voice message announcing "The time to go to the restroom is approaching" was heard. The subject could push the button near the chair to simulate going to the restroom after this suggestion. If the subject ignored the first suggestion, "The time to go to the restroom will be approaching quickly" was announced 5 seconds after the first suggestion. When the subject pushed the button by the chair, the subject cannot bring the shopping basket to the chair in accordance with real shopping situations-shoppers are usually prohibited in bringing shopping items to the restroom before checkout. Therefore, the subject must return the shopping basket to the start position before returning to the chair. After pushing the button, the subject could resume shopping. However, the subject could also ignore the second suggestion.

\section{Toilet Alert}

10 seconds after the subject ignored the second 


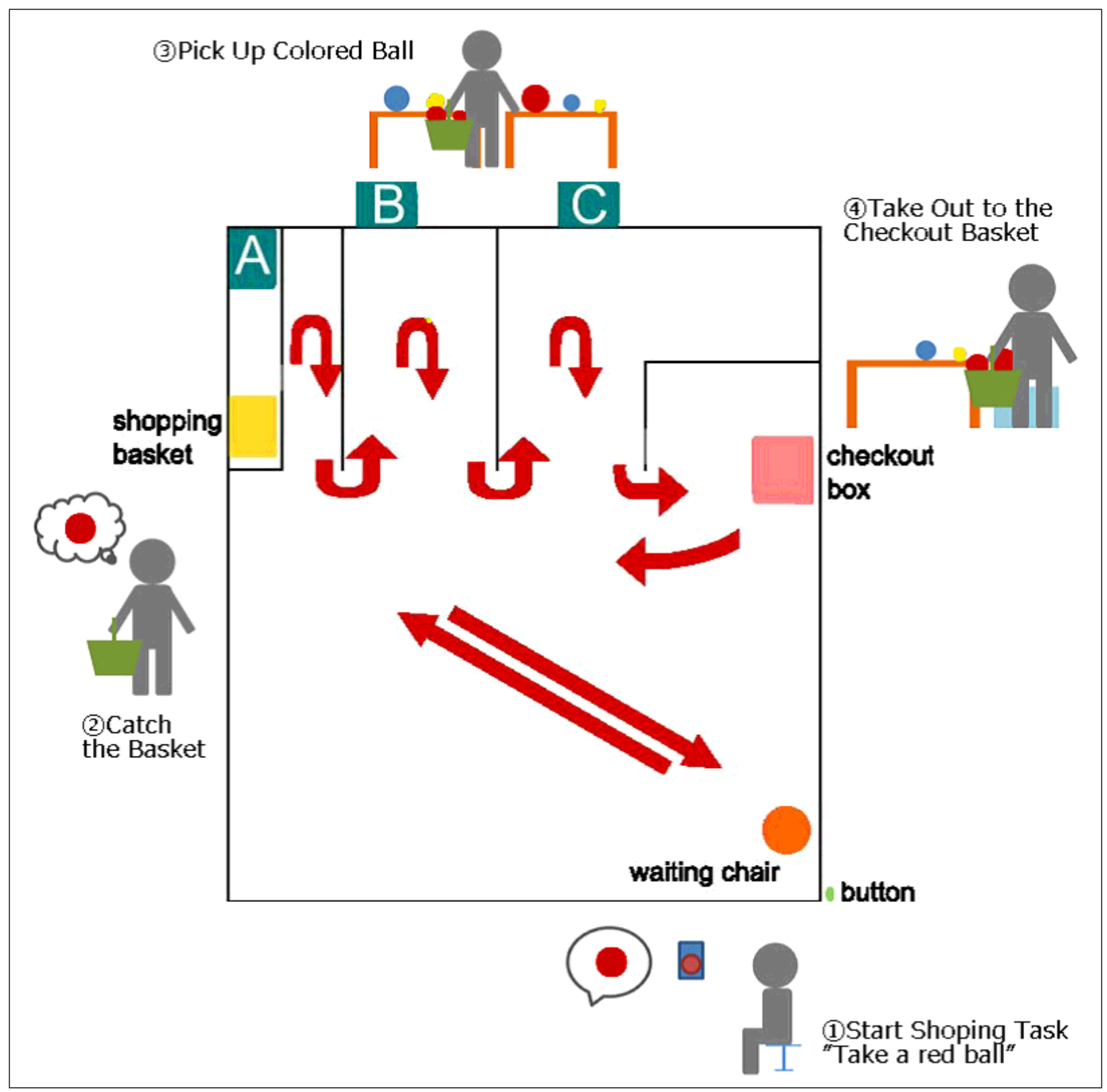

Fig. 1: Experimental Setup

suggestion, a voice message announcing "Go to the restroom now" was heard, along with a beeping sound. The subject had to push the button by the chair as soon as possible. When the subject pushed the button by the chair, they could not bring the shopping basket with them; the shopping basket must be returned to the starting position. The subject could resume shopping after the button was pushed.

\section{EXPERIMENTAL RESULTS}

\section{A. Delays From Suggestions to Actions}

The delay from the suggestion to action was the time elapsed from the first toileting suggestion to the subject pushing the button. Fig. 4 shows the results of elderly subject group's average times under each condition. We performed two-factor ANOVA with the significant level $=0.05$ to analyze each suggestion method's delay time. The ANOVA indicated two within-subject factors ("Consideration" and "Step-byStep"); the results are shown in Tab.III. The results show that our proposed suggestion methods could shorten the times between suggestions and toileting.

TABLE III: ANOVA Analysis of Delay Result

\begin{tabular}{lrrr}
\hline & Considering & Step-by-step & Two-factor interaction \\
\hline \hline $\mathrm{F}$ & 795.322 & 352.371 & 11.473 \\
\hline significant(5\%) & Significantly & Significantly & Significantly \\
\hline
\end{tabular}




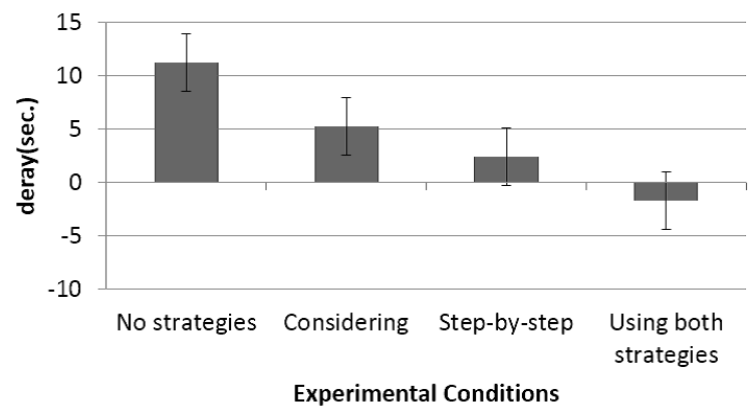

Fig. 4: Experimental Delay Results

\section{B. Shopping Task Achievements}

Fig.5 is the task achievement result. We performed twofactor ANOVA with the significant level $=0.01$ to analyze each suggestion method's shopping task achievements; the results are shown in Tab. IV. The results show that the "Consideration" strategy could improve task performance. On the other hand, the "Step-by-Step" strategy had an adverse effect on the task performance. However, there were some reasons-one was that the time from the first notice until the actual suggestion was very short. The same voice was used for both the first suggestion and the actual notice, causing miscommunication and poor performance.

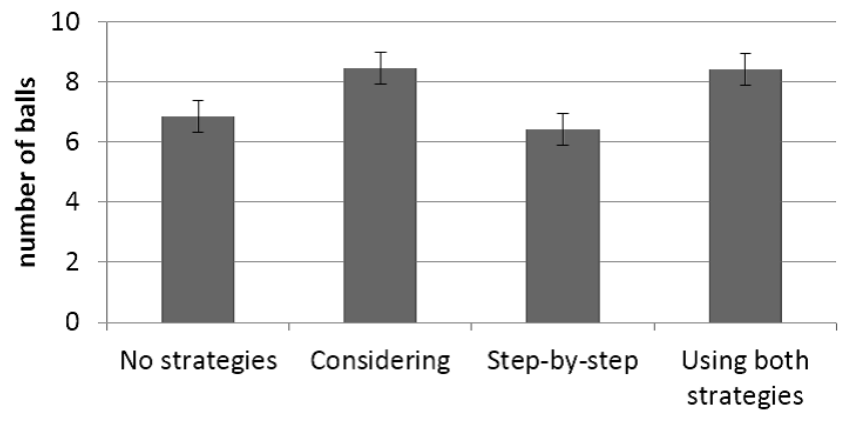

Experimental Conditions

Fig. 5: Experimental Task Results

TABLE IV: ANOVA Analysis of Task Result

\begin{tabular}{lrrr}
\hline & Considering & Step-by-step & Two-factor interaction \\
\hline \hline $\mathrm{F}$ & 1.177 & 79.816 & 0.916 \\
\hline significant(5\%) & No significantly & significantly & No significantly \\
\hline
\end{tabular}

\section{DISCUSSION}

This experiment's two key points are listed below. Both suggestion methods were effective in reducing delays from suggestions to actions.
- Both suggestion methods were effective in reducing delays from suggestions to actions.

- The "Consideration" strategy could improve task performance.

These notifications seem to be enough to achieve our system aims without being ignored and interfering with other tasks. However, the number of completed tasks decreased in the experiments when the "Step-by-Step" notifications were used; it is considered that the time from the first suggestion to the actual notice was very short; the same voice was also used for both, leading to miscommunication and poor performance.

\section{CONCLUSION}

In this paper, we proposed two toilet timing suggestion methods for the elderly in order to support their activities outside the home. For this purpose, the "Consideration" and "Step-by-Step" strategies were considered. Based on the experimental results of the suggestion methods, the delay time from responding to the suggestions to toileting improved by using the proposed methods. As for the two suggestion methods considered, task achievement improved only under the "Consideration" strategy condition. However, this it might have been partly due to improper experimental settings.

\section{ACKNOWLEDGMENTS}

This research is supported by the Ministry of Internal Affairs and Communications of Japan and a Grant-in-Aid for Challenging Exploratory Research from JSPS. We would like to thank Dr. Norihiro Hagita of ATR for the opportunity to conduct this research. This work was partly supported by JSPS KAKENHI Grant Numbers 24300047.

\section{REFERENCES}

[1] KINUKAWA, HAMADA, MIURA, TAKADA: EFFECTS GENERATED BY OUTGOING BEHAVIOR ON THE MENTAL STATE OF THE INSTITUTION-BASED ELDERLY WITH DEMENTIA. Journal of Architectural Institute of Japan,Vol.592,pp.17-24, 2005(in Japanese).

[2] KOMATSU, OKAYAMA, KIMURA: WATER INTAKE OF HOMELIVING ADL-INDEPENDENT ELDERLYPEOPLE : ITS RELATIONSHIPS WITH FACTORS OF THEIR WATER DRINKING BEHAVIOR. Journal of Japan Society of Physiological Anthropology,Vol.9,pp.25-30, 2004(in Japanese).

[3] TSUJI, YONEZAWA, YAMAZOE, ABE, KUWAHARA, MORIMOTO: STUDY OF THE SCHEDULE NOTIFICATION METHOD ON THE SUPPORTING SYSTEM OF LIFE ACTION FOR THE ELDERLY. The 78th Conference of the Human Interface Society, SIG-NOI-06, 2011(in Japanese)

[4] TSUJI, KUWAHARA, MORIMOTO: Study for Hydration Management in Elderly People. Journal of Human Interface Society, Vol.16, No.2,pp.97-102, 2014(in Japanese).

[5] TSUJI, KUWAHARA, MORIMOTO: The Design and Evaluation of the Body Water Management System to Support the Independent Living of the Older Adult. 14th International Conference on Computers Helping People with Special Needs, Saint-Denis, France(July 2014)

[6] ICHIKAWA: Kiso seirigaku. Asakura Publishing Co., Ltd, 1978(in Japanese).

[7] KIMURA, NEGORO: Simple seirigaku[Simple physiological sciences](6th edition). Nankodo Co., Ltd, 2009 (in Japanese).

[8] OKAMOTO: Koreisya ni okeru kaki oyobi touki no suibun suitou[Water balance in the elderly in summer and winter]. Journal of Japanese Society of Biometeorilogy ,Vol.35(1),pp.53-60, 1998(in Japanese). 
[9] Japan Ministry of Education, Culture, Sports, Science and Technology Heisei 22 nen tairyoku undo noryoku cho-sa[Physical fitness and exercise ability investigation 2010]. http://www.estat.go.jp/SG1/estat/Xlsdl.do?sinfid=000012450666 (2014-1-21）

[10] Yorimoto, Shinya, Nakai, Yoshida: Taijyu keisoku kara motometa chukounensya no suibun suitou[Water balance of elderly in summer and winter calculate from body weight measurement]. Journal of Japanese Society of Biometeorilogy ,Vol.45(3),pp.54, 2008(in Japanese). 